\title{
On Skew Circulant Type Matrices Involving Any Continuous Fibonacci Numbers
}

\author{
Zhaolin Jiang, Jinjiang Yao, and Fuliang Lu \\ School of Science, Linyi University, Shuangling Road, Linyi 276005, China \\ Correspondence should be addressed to Jinjiang Yao; yaojinjiang@lyu.edu.cn
}

Received 9 April 2014; Accepted 30 April 2014; Published 18 June 2014

Academic Editor: Zidong Wang

Copyright (C) 2014 Zhaolin Jiang et al. This is an open access article distributed under the Creative Commons Attribution License, which permits unrestricted use, distribution, and reproduction in any medium, provided the original work is properly cited.

Circulant and skew circulant matrices have become an important tool in networks engineering. In this paper, we consider skew circulant type matrices with any continuous Fibonacci numbers. We discuss the invertibility of the skew circulant type matrices and present explicit determinants and inverse matrices of them by constructing the transformation matrices. Furthermore, the maximum column sum matrix norm, the spectral norm, the Euclidean (or Frobenius) norm, and the maximum row sum matrix norm and bounds for the spread of these matrices are given, respectively.

\section{Introduction}

Skew circulant and circulant matrices have important applications in various networks engineering. Joy and Tavsanoglu [1] showed that feedback matrices of ring cellular neural networks, which can be described by the ODE, are block circulants. A special class of the feedback delay network using circulant matrices was proposed [2]. Jing and Jafarkhani [3] proposed distributed differential space-time codes that work for networks with any number of relays using circulant matrices. Exploiting the circulant structure of the channel matrices, Eghbali et al. [4] analysed the realistic near fast fading scenarios with circulant frequency selective channels. Rocchesso [5] presented particular choices of the feedback coefficients, namely, Galois sequences, arranged in a circulant matrix, to produce a maximum echo density in the time response. Sardellitti et al. [6] used an analytical expression for the eigenvalues of a block circulant matrix as a function of the coverage radius. Li et al. [7] gave a low-complexity binary frame-wise network coding encoder design based on circulant matrix. Hirt and Massey [8] introduced discrete time Fourier transform precoding to the proposed multihop relay system involving circulant matrix. When considering a single-input single-output transmission with $\mathrm{CFO}$ and omitting the relay index subscript, Wang et al. [9] proved that the intercarrier interference matrix is a circulant matrix.
The system model of the OFDM based AF relay networks as well as the strategy of the superimposed training involves circulant matrix [10]. Two-way transmission model considered in [11] ensured the circular convolution between two frequency selective channels.

The skew circulant matrices as preconditioners for linear multistep formulae- (LMF-) based ordinary differential equations (ODEs) codes, Hermitian, and skew-Hermitian Toeplitz systems were considered in [12-15]. Lyness and Sørevik employed a skew circulant matrix to construct s-dimensional lattice rules in [16]. Compared with cyclic convolution algorithm, the skew cyclic convolution algorithm [17] was able to perform filtering procedure in approximately half of computational cost for real signals. In [18] two new normalform realizations were presented which utilize circulant and skew circulant matrices as their state transition matrices. The well-known second-order coupled form is a special case of the skew circulant form. Li et al. [19] gave the style spectral decomposition of skew circulant matrix firstly and then dealt with the optimal backward perturbation analysis for the linear system with skew circulant coefficient matrix. In [20], a new fast algorithm for optimal design of block digital filters (BDFs) was proposed based on skew circulant matrix.

Besides, some scholars have given various algorithms for the determinants and inverses of nonsingular circulant matrices. Unfortunately, the computational complexity of 
these algorithms is very amazing huge with the order of matrix increasing. However, some authors gave the explicit determinants and inverses of circulant and skew circulant matrices involving some famous numbers. For example, Yao and Jiang [21] considered the determinants, inverses, norm, and spread of skew circulant type matrices involving any continuous Lucas numbers. Shen et al. considered circulant matrices with Fibonacci and Lucas numbers and presented their explicit determinants and inverses by constructing the transformation matrices [22]. Gao et al. [23] gave explicit determinants and inverses of skew circulant and skew left circulant matrices with Fibonacci and Lucas numbers. Jiang et al. [24, 25] considered the skew circulant and skew left circulant matrices with the $k$-Fibonacci numbers and the $k$-Lucas numbers and discussed the invertibility of the these matrices and presented their determinant and the inverse matrix by constructing the transformation matrices, respectively. Jaiswal evaluated some determinants of circulant whose elements are the generalized Fibonacci numbers [26]. Lind presented the determinants of circulant and skew circulant involving Fibonacci numbers [27]. Dazheng [28] gave the determinant of the Fibonacci-Lucas quasi-cyclic matrices.

Recently, there are several papers on the norms of some special matrices. Solak [29] established the lower and upper bounds for the spectral norms of circulant matrices with classical Fibonacci and Lucas numbers entries. Ípek [30] investigated an improved estimation for spectral norms of these matrices. Shen and Cen [31] gave upper and lower bounds for the spectral norms of $r$-circulant matrices in the form of $A=C_{r}\left(F_{0}, F_{1}, \ldots, F_{n-1}\right), B=C_{r}\left(L_{0}, L_{1}, \ldots, L_{n-1}\right)$, and they also obtained some bounds for the spectral norms of Kronecker and Hadamard products of matrix $A$ and matrix $B$. Akbulak and Bozkurt [32] found upper and lower bounds for the spectral norms of Toeplitz matrices such that $a_{i j} \equiv$ $F_{i-j}$ and $b_{i j} \equiv L_{i-j}$. The convergence in probability and in distribution of the spectral norm of scaled Toeplitz, circulant, reverse circulant, symmetric circulant, and a class of $k$ circulant matrices were discussed in [33].

Beginning with Mirsky [34], several authors [35-37] have obtained bounds for the spread of a matrix.

The Fibonacci sequences are defined by the following recurrence relations $[22,23,26-32]$ :

$$
F_{n+1}=F_{n}+F_{n-1} \quad \text { where } F_{0}=0, F_{1}=1 .
$$

The $\left\{F_{n}\right\}$ is given by the formula

$$
F_{n}=\frac{\alpha^{n}-\beta^{n}}{\alpha-\beta},
$$

where $\alpha$ and $\beta$ are the roots of the characteristic equation $x^{2}-$ $x-1=0$.

The Fibonacci sequences were introduced for the first time by the famous Italian mathematician Leonardo of Pisa (nicknamed Fibonacci). It is well known that the ratio of two consecutive classical Fibonacci numbers converges to the golden mean, or the golden section, $(1+\sqrt{5}) / 2$, which appears in modern research in many fields from architecture $[38,39]$ to physics of high energy particles [40]. As is shown in [41, 42], the hyperbolic Fibonacci functions can lead to creation of the Lobachevsky Fibonacci and Minkovsky Fibonacci geometry which are of great importance for theoretical physics. In the 19th century the French mathematician Francois Edouard Anatole Lucas (1842-1891) introduced the so-called Lucas numbers given by the recursive relation $L_{n}=L_{n-1}+L_{n-2}, n \geq$ 2 , with the seeds $L_{0}=2$ and $L_{1}=1$. The determinants, inverses, norm, and spread of skew circulant type matrices involving any continuous Lucas numbers are considered in [21].

The purpose of this paper is to obtain the explicit determinants, explicit inverses, norm, and spread of skew circulant type matrices involving any continuous Fibonacci numbers. And we generalize the result [23]. In passing, the norm and spread of skew circulant type matrices have not been research. It is hoped that this paper will help in changing this.

In the following, let $r$ be a nonnegative integer. We adopt the following two conventions $0^{0}=1$ and, for any sequence $\left\{a_{n}\right\}, \sum_{k=i}^{n} a_{k}=0$ in case $i>n$.

Definition 1 (see [21]). A skew circulant matrix with the first row $\left(a_{1}, a_{2}, \ldots, a_{n}\right)$ is meant to be a square matrix of the form

$$
\left(\begin{array}{ccccc}
a_{1} & a_{2} & \ldots & a_{n-1} & a_{n} \\
-a_{n} & a_{1} & a_{2} & \ldots & a_{n-1} \\
\vdots & -a_{n} & a_{1} & \ddots & \vdots \\
-a_{3} & \vdots & \ddots & \ddots & a_{2} \\
-a_{2} & -a_{3} & \ldots & -a_{n} & a_{1}
\end{array}\right)_{n \times n},
$$

denoted by $\operatorname{SCirc}\left(a_{1}, a_{2}, \ldots, a_{n}\right)$.

Definition 2 (see [21]). A skew left circulant matrix with the first row $\left(a_{1}, a_{2}, \ldots, a_{n}\right)$ is meant to be a square matrix of the form

$$
\left(\begin{array}{ccccc}
a_{1} & a_{2} & a_{3} & \ldots & a_{n} \\
a_{2} & a_{3} & \ldots & a_{n} & -a_{1} \\
a_{3} & . & . & . & \vdots \\
\vdots & a_{n} & -a_{1} & \ldots & -a_{n-2} \\
a_{n} & -a_{1} & \ldots & -a_{n-2} & -a_{n-1}
\end{array}\right)_{n \times n},
$$

denoted by $\operatorname{SLCirc}\left(a_{1}, a_{2}, \ldots, a_{n}\right)$.

Lemma 3 (see $[30,31]$ ). Let $\left\{F_{n}\right\}$ be Fibonacci numbers; then,

(i) $\sum_{i=0}^{n-1} F_{i}=F_{n+1}-1$,

(ii) $\sum_{i=0}^{n-1} F_{i}^{2}=F_{n} F_{n-1}$,

(iii) $\sum_{i=0}^{n-1} i F_{i}=(n-1) F_{n+1}-F_{n+2}+2$. 


\section{Determinant and Inverse of Skew Circulant Matrix with the Fibonacci Numbers}

In this section, let $B_{r, n}=\operatorname{SCirc}\left(F_{r+1}, \ldots, F_{r+n}\right)$ be a skew circulant matrix. Firstly, we give a determinant explicit formula for the matrix $B_{r, n}$. Afterwards, we prove that $B_{r, n}$ is an invertible matrix for $n \geq 2$, and then we find the inverse of the matrix $B_{r, n}$. In the following, let

$$
\begin{gathered}
x=-\frac{F_{r}+F_{r+n}}{F_{r+1}+F_{r+n+1}}, \quad s=\frac{F_{r+2}}{F_{r+1}}, \\
b=F_{r+1}+F_{r+n+1}, \quad a=F_{r}+F_{r+n}, \\
f_{n}=F_{r+1}+s F_{r+n} \\
+\sum_{k=1}^{n-2}\left(s F_{r+k+1}-F_{r+k+2}\right) \cdot x^{n-(k+1)}, \\
f_{n^{\prime}}=\sum_{k=1}^{n-1} F_{r+k+1} \cdot x^{n-(k+1)} .
\end{gathered}
$$

Theorem 4. Let $B_{r, n}=\operatorname{SCirc}\left(F_{r+1}, \ldots, F_{r+n}\right)$ be a skew circulant matrix; then,

$$
\operatorname{det} B_{r, n}=F_{r+1} \cdot f_{n} \cdot b^{n-2},
$$

where $F_{r+n}$ is the $(r+n)$ th Fibonacci number. In particular, when $r=0$, we get the result of [23].

Proof. Obviously, det $B_{r, 2}=F_{r+1}^{2}+F_{r+2}^{2}$ satisfies the equation. In case $n>2$, let

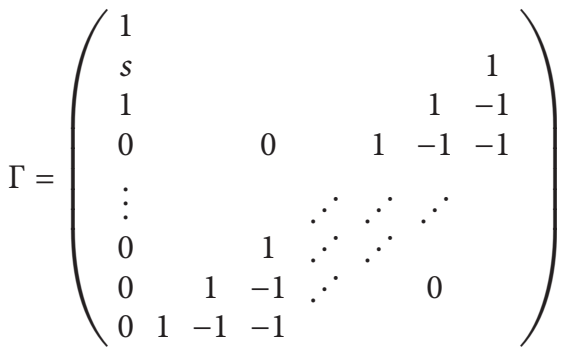

$$
\begin{aligned}
& \Pi_{1}=\left(\begin{array}{cccccc}
1 & 0 & 0 & \cdots & 0 & 0 \\
0 & x^{n-2} & 0 & \cdots & 0 & 1 \\
0 & x^{n-3} & 0 & \cdots & 1 & 0 \\
\vdots & \vdots & \vdots & \ddots & \vdots & \vdots \\
0 & x & 1 & \cdots & 0 & 0 \\
0 & 1 & 0 & \cdots & 0 & 0
\end{array}\right)
\end{aligned}
$$

be two $n \times n$ matrices; then, we have

$$
\Gamma B_{r, n} \Pi_{1}=\left(\begin{array}{cccccc}
F_{r+1} & f_{n}^{\prime} & b_{13} & \cdots & b_{1(n-1)} & b_{1 n} \\
0 & f_{n} & b_{23} & \cdots & b_{2(n-1)} & b_{2 n} \\
0 & 0 & b & & & \\
0 & 0 & a & \ddots & & \\
\vdots & \vdots & & \ddots & b & \\
0 & 0 & & & a & b
\end{array}\right) \text {, }
$$

where

$$
\begin{aligned}
& b_{1 j}=F_{r+n+2-j}, \\
& b_{2 j}=s F_{r+n+2-j}-F_{r+n+3-j}, \quad(j=3,4, \ldots, n) .
\end{aligned}
$$

So it holds that

$$
\begin{aligned}
& \operatorname{det} \Gamma \operatorname{det} B_{r, n} \operatorname{det} \Pi_{1} \\
& =F_{r+1} \cdot f_{n} \cdot b^{n-2} \\
& =F_{r+1}\left[F_{r+1}+s F_{r+n}\right. \\
& \left.\quad+\sum_{k=1}^{n-2}\left(s F_{r+1+k}-F_{r+2+k}\right) \cdot x^{n-(k+1)}\right] \\
& \quad \times\left(F_{r+1}+F_{r+n+1}\right)^{n-2},
\end{aligned}
$$

while taking $\operatorname{det} \Gamma=\operatorname{det} \Pi_{1}=(-1)^{(n-1)(n-2) / 2}$, we can get

$$
\begin{aligned}
\operatorname{det} B_{r, n}= & F_{r+1}\left[\frac{F_{r+2}}{F_{r+1}} \cdot F_{r+n}+F_{r+1}\right. \\
& \left.\quad+\sum_{k=1}^{n-2}\left(\frac{F_{r+2}}{F_{r+1}} \cdot F_{r+1+k}-F_{r+2+k}\right) \cdot x^{n-(k+1)}\right] \\
& \times\left(F_{r+1}+F_{r+n+1}\right)^{n-2} .
\end{aligned}
$$

This completes the proof.

Theorem 5. Let $B_{r, n}=\operatorname{SCirc}\left(F_{r+1}, \ldots, F_{r+n}\right)$ be a skew circulant matrix; then, $B_{r, n}$ is an invertible matrix. Specially, when $r=0$, we get the result of [23].

Proof. Taking $n=2$ in Theorem 4 , we have $\operatorname{det} B_{r, 2}=F_{r+1}^{2}+$ $F_{r+2}^{2} \neq 0$. Hence $B_{r, 2}$ is invertible. In case $n>2$, since $F_{r+n}=$ $\left(\alpha^{r+n}-\beta^{r+n}\right) /(\alpha-\beta)$, where $\alpha+\beta=1, \alpha \beta=-1$, we obtain

$$
\begin{aligned}
& f\left(\omega^{k} \eta\right) \\
& =\sum_{j=1}^{n} F_{r+j}\left(\omega^{k} \eta\right)^{j-1} \\
& =\frac{1}{\alpha-\beta} \sum_{j=1}^{n}\left(\alpha^{r+j}-\beta^{r+j}\right)\left(\omega^{k} \eta\right)^{j-1} \\
& =\frac{1}{\alpha-\beta}\left[\frac{\alpha^{r+1}\left(1+\alpha^{n}\right)}{1-\alpha \omega^{k} \eta}-\frac{\beta^{r+1}\left(1+\beta^{n}\right)}{1-\beta \omega^{k} \eta}\right]
\end{aligned}
$$




$$
\begin{gathered}
=\frac{1}{\alpha-\beta}\left[\frac{\left(\alpha^{r+1}-\beta^{r+1}\right)+\left(\alpha^{r+n+1}-\beta^{r+n+1}\right)}{1-(\alpha+\beta) \omega^{k} \eta+\alpha \beta \omega^{2 k} \eta^{2}}\right. \\
\left.-\frac{\alpha \beta\left(\alpha^{r}-\beta^{r}+\alpha^{r+n}-\beta^{r+n}\right) \omega^{k} \eta}{1-(\alpha+\beta) \omega^{k} \eta+\alpha \beta \omega^{2 k} \eta^{2}}\right] \\
=\frac{F_{r+1}+F_{r+n+1}+\left(F_{r}+F_{r+n}\right) \omega^{k} \eta}{1-\omega^{k} \eta-\omega^{2 k} \eta^{2}} \\
(k=1,2, \ldots, n-1),
\end{gathered}
$$

where $\omega=\exp (2 \pi i / n), \eta=\exp (\pi i / n)$. If there exists $\omega^{l} \eta(l=$ $1,2, \ldots, n-1)$ such that $f\left(\omega^{l} \eta\right)=0$, we obtain $F_{r+1}+F_{r+n+1}+$ $\left(F_{r}+F_{r+n}\right) \omega^{k} \eta=0$ for $1-\omega^{l} \eta-\omega^{2 l} \eta^{2} \neq 0$, and hence it follows that $\omega^{l} \eta=-\left(F_{r+1}+F_{r+n+1}\right) /\left(F_{r}+F_{r+n}\right)$ is a real number. Since

$$
\begin{aligned}
\omega^{l} \eta & =\exp \left(\frac{(2 l+1) \pi i}{n}\right) \\
& =\cos \frac{(2 l+1) \pi}{n}+i \sin \frac{(2 l+1) \pi}{n}
\end{aligned}
$$

it yields that $\sin ((2 l+1) \pi / n)=0$, so we have $\omega^{l} \eta=-1$ for $0<(2 l+1) \pi / n<2 \pi$. Since $x=-1$ is not the root of the equation,

$$
F_{r+1}+F_{r+n+1}+\left(F_{r}+F_{r+n}\right) x=0, \quad(n>2) .
$$

We obtain $f\left(\omega^{k} \eta\right) \neq 0$ for any $\omega^{k} \eta(k=1,2, \ldots, n-1)$, while

$$
\begin{aligned}
f(\eta) & =\sum_{j=1}^{n} F_{r+j} \eta^{j-1} \\
& =\frac{F_{r+1}+F_{r+n+1}+\left(F_{r}+F_{r+n}\right) \eta}{1-\eta-\eta^{2}} \neq 0 .
\end{aligned}
$$

It follows from Lemma 3 in [21] that the conclusion holds.

Lemma 6. Let the matrix $\mathscr{G}=\left[g_{i, j}\right]_{i, j=1}^{n-2}$ be of the form

$$
g_{i j}= \begin{cases}F_{r+1}+F_{r+n+1}, & i=j, \\ F_{r}+F_{r+n}, & i=j+1, \\ 0, & \text { otherwise. }\end{cases}
$$

Then the inverse $\mathscr{G}^{-1}=\left[g_{i, j}^{\prime}\right]_{i, j=1}^{n-2}$ of $\mathscr{G}$ is equal to

$$
g_{i, j}^{\prime}= \begin{cases}\frac{\left[-\left(F_{r}+F_{r+n}\right)\right]^{i-j}}{\left(F_{r+1}+F_{r+n+1}\right)^{i-j+1}}, & i \geq j, \\ 0, & i<j .\end{cases}
$$

In particular, when $r=0$, we get the result of [23].

Proof. Let $c_{i j}=\sum_{k=1}^{n-2} g_{i k} g_{k j}^{\prime}$.
Then $c_{i, j}=0$, for $i<j, c_{i i}=g_{i i} g_{i i}^{\prime}=\left(F_{r+1}+F_{r+n+1}\right)$. $1 /\left(F_{r+1}+F_{r+n+1}\right)=1$, for $i=j$, and

$$
\begin{aligned}
c_{i j}= & \sum_{k=1}^{n-2} g_{i k} g_{k j}^{\prime}=g_{i, i-1} g_{i-1, j}^{\prime}+g_{i, i} g_{i, j}^{\prime} \\
= & \left(F_{r}+F_{r+n}\right) \cdot \frac{\left[-\left(F_{r}+F_{r+n}\right)\right]^{i-j-1}}{\left(F_{r+1}+F_{r+n+1}\right)^{i-j}} \\
& +\left(F_{r+1}+F_{r+n+1}\right) \cdot \frac{\left[-\left(F_{r}+F_{r+n}\right)\right]^{i-j}}{\left(F_{r+1}+F_{r+n+1}\right)^{i-j+1}}
\end{aligned}
$$

$$
=0 \text {, }
$$

for $i \geq j+1$

Hence, we get $\mathscr{G} \mathscr{G}^{-1}=I_{n-2}$, where $I_{n-2}$ is an $(n-2) \times(n-2)$ identity matrix. Similarly, we can verify $\mathscr{G}^{-1} \mathscr{G}=I_{n-2}$. Thus, the proof is completed.

Theorem 7. Let $B_{r, n}=\operatorname{SCirc}\left(F_{r+1}, \ldots, F_{r+n}\right)$ be a skew circulant matrix; then,

$$
B_{r, n}^{-1}=\frac{1}{f_{n}} \cdot \operatorname{SCirc}\left(x_{1}^{\prime}, x_{2}^{\prime}, \ldots, x_{n}^{\prime}\right),
$$

where

$$
\begin{aligned}
& x_{1}^{\prime}=-\left(F_{r+3}-s F_{r+2}\right) \cdot \frac{(-a)^{n-3}}{b^{n-1}} \\
&-\sum_{i=1}^{n-3}\left(F_{r+n+2-i}-s F_{r+n+1-i}\right) \cdot \frac{(-a)^{i-1}}{b^{i}}, \\
& x_{2}^{\prime}=-s-\sum_{i=1}^{n-2}\left(F_{r+n+1-i}-s F_{r+n-i}\right) \cdot \frac{(-a)^{i-1}}{b^{i}}, \\
& x_{3}^{\prime}=-\left(F_{r+3}-s F_{r+2}\right) \cdot \frac{1}{b}, \\
& x_{4}^{\prime}=-\sum_{i=1}^{2}\left(F_{r+1+i}-s F_{r+i}\right) \cdot \frac{(-a)^{i-1}}{b^{i}}, \\
& x_{k}^{\prime}=-\frac{1}{f_{n}} \sum_{i=1}^{2}\left(F_{r+1+i}-s F_{r+i}\right) \cdot \frac{(-a)^{k-5+i}}{b^{k-4+i}}, \\
&(k=5,6, \ldots, n) .
\end{aligned}
$$

In particular, when $r=0$, we get the result of [23].

Proof. Let

$$
\Pi_{2}=\left(\begin{array}{cccccc}
1 & -\frac{f_{n}^{\prime}}{F_{r+1}} & \pi_{13} & \pi_{14} & \cdots & \pi_{1 n} \\
0 & 1 & \pi_{23} & \pi_{24} & \cdots & \pi_{2 n} \\
0 & 0 & 1 & 0 & \cdots & 0 \\
0 & 0 & 0 & 1 & \cdots & 0 \\
\vdots & \vdots & \vdots & \vdots & \ddots & \vdots \\
0 & 0 & 0 & 0 & \cdots & 1
\end{array}\right),
$$


where

$$
\begin{gathered}
\pi_{1 j}=\frac{1}{F_{r+1}}\left[\frac{f_{n}^{\prime}}{f_{n}}\left(s F_{r+n+2-j}-F_{r+n+3-j}\right)\right. \\
\left.-F_{r+n+2-j}\right], \quad(j=3,4, \ldots, n), \\
\pi_{2 j}=-\frac{s F_{r+n+2-j}-F_{r+n+3-j}}{f_{n}}, \quad(j=3,4, \ldots, n) .
\end{gathered}
$$

Then we have

$$
\Gamma B_{r, n} \Pi_{1} \Pi_{2}=\left(\begin{array}{cccccc}
F_{r+1} & 0 & 0 & 0 & \cdots & 0 \\
0 & f_{n} & 0 & 0 & \cdots & 0 \\
0 & 0 & b & 0 & \cdots & 0 \\
0 & 0 & a & b & \cdots & 0 \\
\vdots & \vdots & \vdots & \vdots & \ddots & \vdots \\
0 & 0 & 0 & 0 & \cdots & b
\end{array}\right) .
$$

$\Gamma B_{r, n} \Pi_{1} \Pi_{2}=D_{1} \oplus \mathscr{G}, D_{1}=\operatorname{diag}\left(F_{r+1}, f_{n}\right)$ is a diagonal matrix, and $\mathscr{D}_{1} \oplus \mathscr{G}$ is the direct sum of $\mathscr{D}_{1}$ and $\mathscr{G}$. If we denote $\Pi=$ $\Pi_{1} \Pi_{2}$, then we obtain $B_{r, n}^{-1}=\Pi\left(\mathscr{D}_{1}^{-1} \oplus \mathscr{G}^{-1}\right) \Gamma$.

Since the last row elements of the matrix $\Pi$ are $\left(0,1, \pi_{23}, \pi_{24}, \ldots, \pi_{2(n-1)}, \pi_{2 n}\right)$, then the last row elements of the matrix $\Pi\left(\mathscr{D}_{1}^{-1} \oplus \mathscr{G}^{-1}\right)$ are $\left(0,1 / f_{n}, U_{23}, U_{24}, \ldots, U_{2 n}\right)$, where

$$
\begin{array}{r}
U_{23}=\sum_{i=1}^{n-2} \pi_{2(2+i)} \cdot \frac{(-a)^{i-1}}{b^{i}}, \\
U_{2 k}=\sum_{i=1}^{n+1-k} \pi_{2(k-1+i)} \cdot \frac{(-a)^{i-1}}{b^{i}}, \\
\quad(k=3,4, \ldots, n) .
\end{array}
$$

Hence it follows from Lemma 6 that letting $B_{r, n}^{-1}=$ $\operatorname{SCirc}\left(x_{1}, x_{2}, \ldots, x_{n}\right)$ then its last row elements are $\left(-x_{2},-x_{3}, \ldots,-x_{n}, x_{1}\right)$ which are given by the following equations:

$$
\begin{aligned}
-x_{2} & =\frac{s}{f_{n}}+U_{23}=\frac{s}{f_{n}}+\sum_{i=1}^{n-2} \pi_{2(2+i)} \cdot \frac{(-a)^{i-1}}{b^{i}} \\
& =\frac{s}{f_{n}}+\frac{1}{f_{n}} \cdot \sum_{i=1}^{n-2}\left(F_{r+n+1-i}-s F_{r+n-i}\right) \cdot \frac{(-a)^{i-1}}{b^{i}}, \\
-x_{3} & =U_{2 n}=\frac{1}{f_{n}} \cdot \frac{F_{r+3}-s F_{r+2}}{b}
\end{aligned}
$$

$$
\begin{aligned}
& -x_{4}=U_{2(n-1)}-U_{2 n}=\frac{1}{f_{n}}\left[\left(F_{r+2}-s F_{r+1}\right) \cdot \frac{1}{b}\right. \\
& \left.+\left(F_{r+3}-s F_{r+2}\right) \cdot \frac{-a}{b^{2}}\right] \\
& =\frac{1}{f_{n}} \sum_{i=1}^{2}\left(F_{r+1+i}-s F_{r+i}\right) \cdot \frac{(-a)^{i-1}}{b^{i}}, \\
& -x_{k}=U_{2(k-1)}-U_{2 k}-U_{2(k+1)} \\
& =\frac{1}{f_{n}}\left[\left(F_{r+2}-s F_{r+1}\right) \cdot \frac{(-a)^{k-4}}{b^{k-3}}\right. \\
& \left.+\left(F_{r+3}-s F_{r+2}\right) \cdot \frac{(-a)^{k-3}}{b^{k-2}}\right] \\
& =\frac{1}{f_{n}} \sum_{i=1}^{2}\left(F_{r+1+i}-s F_{r+i}\right) \cdot \frac{(-a)^{k-5+i}}{b^{k-4+i}}, \\
& \vdots \\
& -x_{n}=U_{23}-U_{24}-U_{25} \\
& =\frac{1}{f_{n}} \sum_{i=1}^{n-2}\left(F_{r+n+1-i}-s F_{r+n-i}\right) \cdot \frac{(-a)^{i-1}}{b^{i}} \\
& -\frac{1}{f_{n}} \sum_{i=1}^{n-3}\left(F_{r+n-i}-s F_{r+n-1-i}\right) \cdot \frac{(-a)^{i-1}}{b^{i}} \\
& -\frac{1}{f_{n}} \sum_{i=1}^{n-1}\left(F_{r+n-1-i}-s F_{r+n-2-i}\right) \cdot \frac{(-a)^{i-1}}{b^{i}} \\
& =\frac{1}{f_{n}}\left[\left(F_{r+2}-s F_{r+1}\right) \cdot \frac{(-a)^{n-4}}{b^{n-3}}\right. \\
& \left.+\left(F_{r+3}-s F_{r+2}\right) \cdot \frac{(-a)^{n-3}}{b^{n-2}}\right] \\
& =\frac{1}{f_{n}} \sum_{i=1}^{2}\left(F_{r+1+i}-s F_{r+i}\right) \cdot \frac{(-a)^{n-5+i}}{b^{n-4+i}}, \\
& x_{1}=\frac{1}{f_{n}}-U_{23}-U_{24} \\
& =\frac{1}{f_{n}}-\frac{1}{f_{n}} \sum_{i=1}^{n-2}\left(F_{r+n+1-i}-s F_{r+n-i}\right) \cdot \frac{(-a)^{i-1}}{b^{i}} \\
& -\frac{1}{f_{n}} \sum_{i=1}^{n-3}\left(F_{r+n+2-i}-s F_{r+n+1-i}\right) \cdot \frac{(-a)^{i-1}}{b^{i}} \\
& =\frac{1}{f_{n}}\left[1-\left(F_{r+3}-s F_{r+2}\right) \cdot \frac{(-a)^{n-3}}{b^{n-2}}\right. \\
& \left.-\sum_{i=1}^{n-3}\left(F_{r+n+2-i}-s F_{r+n+1-i}\right) \cdot \frac{(-a)^{i-1}}{b^{i}}\right] .
\end{aligned}
$$


Hence, we obtain

$$
\begin{aligned}
& x_{1}=\frac{1}{f_{n}}\left[1-\left(F_{r+3}-s F_{r+2}\right) \cdot \frac{(-a)^{n-3}}{b^{n-1}}\right. \\
& \left.-\sum_{i=1}^{n-3}\left(F_{r+n+2-i}-s F_{r+n+1-i}\right) \cdot \frac{(-a)^{i-1}}{b^{i}}\right], \\
& x_{2}=-\frac{s}{f_{n}}-\frac{1}{f_{n}} \sum_{i=1}^{n-2}\left(F_{r+n+1-i}-s F_{r+n-i}\right) \cdot \frac{(-a)^{i-1}}{b^{i}} \text {, } \\
& x_{3}=-\frac{1}{f_{n}} \cdot \frac{F_{r+3}-s F_{r+2}}{b} \text {, } \\
& x_{4}=-\frac{1}{f_{n}} \sum_{i=1}^{2}\left(F_{r+1+i}-s F_{r+i}\right) \cdot \frac{(-a)^{i-1}}{b^{i}} \text {, } \\
& x_{k}=-\frac{1}{f_{n}} \sum_{i=1}^{2}\left(F_{r+1+i}-s F_{r+i}\right) \cdot \frac{(-a)^{k-5+i}}{b^{k-4+i}} \text {, } \\
& \vdots \\
& x_{n}=-\frac{1}{f_{n}} \sum_{i=1}^{2}\left(F_{r+1+i}-s F_{r+i}\right) \cdot \frac{(-a)^{n-5+i}}{b^{n-4+i}} \text {, } \\
& B_{r, n}^{-1}=\frac{1}{f_{n}} \cdot \operatorname{SCirc}\left(x_{1}^{\prime}, x_{2}^{\prime}, \ldots, x_{n}^{\prime}\right) \text {, }
\end{aligned}
$$

where

$$
\begin{aligned}
& x_{1}^{\prime}=-\left(F_{r+3}-s F_{r+2}\right) \cdot \frac{(-a)^{n-3}}{b^{n-1}} \\
&-\sum_{i=1}^{n-3}\left(F_{r+n+2-i}-s F_{r+n+1-i}\right) \cdot \frac{(-a)^{i-1}}{b^{i}}, \\
& x_{2}^{\prime}=-s-\sum_{i=1}^{n-2}\left(F_{r+n+1-i}-s F_{r+n-i}\right) \cdot \frac{(-a)^{i-1}}{b^{i}}, \\
& x_{3}^{\prime}=-\left(F_{r+3}-s F_{r+2}\right) \cdot \frac{1}{b}, \\
& x_{4}^{\prime}=-\sum_{i=1}^{2}\left(F_{r+1+i}-s F_{r+i}\right) \cdot \frac{(-a)^{i-1}}{b^{i}}, \\
& x_{k}^{\prime}=-\sum_{i=1}^{2}\left(F_{r+1+i}-s F_{r+i}\right) \cdot \frac{(-a)^{k-5+i}}{b^{k-4+i}}, \\
& \quad(k=5,6, \ldots, n) .
\end{aligned}
$$

\section{Norm and Spread of Skew Circulant Matrix with the Fibonacci Numbers}

Theorem 8. Let $B_{r, n}=\operatorname{SCirc}\left(F_{r+1}, \ldots, F_{r+n}\right)$ be a skew circulant matrix; then three kinds of norms of $B_{r, n}$ are given by

$$
\begin{aligned}
\left\|B_{r, n}\right\|_{1} & =\left\|B_{r, n}\right\|_{\infty}=F_{r+n+2}-F_{r+2}, \\
\left\|B_{r, n}\right\|_{F} & =\sqrt{n\left(F_{r+n} F_{r+n+1}-F_{r} F_{r+1}\right)} .
\end{aligned}
$$

Proof. By Definition 8 in [21] and (5), we have

$$
\left\|B_{r, n}\right\|_{1}=\left\|B_{r, n}\right\|_{\infty}=\sum_{i=1}^{n} F_{r+i}=F_{r+n+2}-F_{r+2} .
$$

According to Definition 8 in [21] and (6), we know

$$
\begin{aligned}
\left(\left\|B_{r, n}\right\|_{F}\right)^{2} & =\sum_{i=1}^{n} \sum_{j=1}^{n}\left|a_{i j}\right|^{2} \\
& =n \sum_{i=1}^{n} F_{r+i}^{2} \\
& =n\left(\sum_{i=0}^{r+n} F_{i}^{2}-\sum_{i=0}^{r} F_{i}^{2}\right) \\
& =n\left(F_{r+n} F_{r+n+1}-F_{r} F_{r+1}\right) .
\end{aligned}
$$

Thus

$$
\left\|B_{r, n}\right\|_{F}=\sqrt{n\left(F_{r+n} F_{r+n+1}-F_{r} F_{r+1}\right)} .
$$

Theorem 9. Let

$$
B_{r, n}^{\prime}=\operatorname{SCirc}\left(F_{r+1},-F_{r+2}, \ldots,-F_{r+n-1}, F_{r+n}\right)
$$

be an odd-order alternative skew circulant matrix and let $n$ be odd. Then

$$
\left\|B_{r, n}^{\prime}\right\|_{2}=\sum_{i=1}^{n} F_{r+i}=F_{r+n+2}-F_{r+2} .
$$

Proof. By Lemma 3 in [21], we have

$$
\lambda_{j}\left(B_{r, n}^{\prime}\right)=\sum_{i=1}^{n}(-1)^{i-1} F_{r+i}\left(\omega^{j} \eta\right)^{i-1}
$$

So

$$
\begin{aligned}
\left|\lambda_{j}\left(B_{r, n}^{\prime}\right)\right| & \leq \sum_{i=1}^{n}\left|(-1)^{i-1} F_{r+i}\right| \cdot\left|\left(\omega^{j} \eta\right)^{i-1}\right| \\
& =\sum_{i=1}^{n} F_{r+i},
\end{aligned}
$$

This completes the proof.

for all $j=0,1, \ldots, n-1$. 
Since $n$ is odd, $\sum_{i=1}^{n} F_{r+i}$ is an eigenvalue of $B_{r, n}^{\prime}$; that is

$$
\begin{gathered}
\left(\begin{array}{cccc}
F_{r+1} & -F_{r+2} & \cdots & F_{r+n} \\
-F_{r+n} & F_{r+1} & \cdots & -F_{r+n-1} \\
\vdots & \vdots & \ddots & \vdots \\
F_{r+2} & -F_{r+3} & \cdots & F_{r+1}
\end{array}\right)\left(\begin{array}{c}
1 \\
-1 \\
1 \\
-1 \\
\vdots \\
1
\end{array}\right) \\
=\sum_{i=1}^{n} F_{r+i} \cdot\left(\begin{array}{c}
1 \\
-1 \\
1 \\
-1 \\
\vdots \\
1
\end{array}\right) .
\end{gathered}
$$

To sum up, we can get

$$
\max _{0 \leq j \leq n-1}\left|\lambda_{j}\left(B_{r, n}^{\prime}\right)\right|=\sum_{i=1}^{n} F_{r+i} .
$$

Since all skew circulant matrices are normal, by Lemma 9 in [21], (5), and (41), we obtain

$$
\left\|B_{r, n}^{\prime}\right\|_{2}=\sum_{i=1}^{n} F_{r+i}=F_{r+n+2}-F_{r+2},
$$

which completes the proof.

Theorem 10. Let $B_{r, n}=\operatorname{SCirc}\left(F_{r+1}, \ldots, F_{r+n}\right)$ be a skew circulant matrix; then, the bounds for the spread of $B_{r, n}$ are

$$
\begin{aligned}
& s\left(B_{r, n}\right) \leqslant \sqrt{2 n\left(F_{r+n} F_{r+n+1}-F_{r+1} F_{r+2}\right)}, \\
& s\left(B_{r, n}\right) \geq \frac{1}{n-1}\left|2 F_{r+n+4}-n F_{r+n+2}-n F_{r+3}-2 F_{r+4}\right| .
\end{aligned}
$$

Proof. The trace of $B_{r, n}$ is denoted by $\operatorname{tr} B_{r, n}=n F_{r+1}$. By (18) in [21] and (32), we know

$$
s\left(B_{r, n}\right) \leqslant \sqrt{2 n\left(F_{r+n} F_{r+n+1}-F_{r+1} F_{r+2}\right)} .
$$

Since

$$
\begin{aligned}
\sum_{i \neq j} a_{i j}= & \sum_{k=2}^{n}(n-(k-1)) F_{r+k}-\sum_{k=2}^{n}(k-1) F_{r+k} \\
= & (n+2) \sum_{k=2}^{n} F_{r+k}-2 \sum_{k=2}^{n} k F_{r+k} \\
= & (n+2)\left(F_{r+n+2}-F_{r+3}\right) \\
& -2\left[\sum_{k=2}^{n}(r+k) F_{r+k}-\sum_{k=2}^{n} r F_{r+k}\right],
\end{aligned}
$$

furthermore, by (5) and (7),

$$
\begin{aligned}
& \sum_{i \neq j} a_{i j}=(n+2)\left(F_{r+n+2}-F_{r+3}\right) \\
&-2\left[(r+n) F_{r+n+2}-F_{r+n+3}-(r+1) F_{r+3}\right. \\
&\left.\quad+F_{r+4}-r F_{r+n+2}+r F_{r+3}\right] \\
&=2 F_{r+n+4}-n F_{r+n+2}-n F_{r+3}-2 F_{r+4} .
\end{aligned}
$$

By (19) in [21], we have

$$
s\left(B_{r, n}\right) \geq \frac{1}{n-1}\left|2 F_{r+n+4}-n F_{r+n+2}-n F_{r+3}-2 F_{r+4}\right| .
$$

\section{Determinant and Inverse of Skew Left Circulant Matrix with the Fibonacci Numbers}

In this section, let $B_{r, n}^{\prime \prime}=\operatorname{SLCirc}\left(F_{r+1}, \ldots, F_{r+n}\right)$ be a skew left circulant matrix. By using the obtained conclusions in Section 2, we give a determinant explicit formula for the matrix $B_{r, n}^{\prime \prime}$. Afterwards, we prove that $B_{r, n}^{\prime \prime}$ is an invertible matrix for any positive interger $n$. The inverse of the matrix $B_{r, n}^{\prime \prime}$ is also presented.

According to Lemma 5 in [21], Lemma 6 in [21], and Theorems 4, 5, and 7, we can obtain the following theorems.

Theorem 11. Let $B_{r, n}^{\prime \prime}=\operatorname{SLCirc}\left(F_{r+1}, \ldots, F_{r+n}\right)$ be a skew left circulant matrix; then,

$\operatorname{det} B_{r, n}^{\prime \prime}$

$$
\begin{aligned}
& =(-1)^{n(n-1) / 2} F_{r+1}\left[\frac{F_{r+2}}{F_{r+1}} \cdot F_{r+n}+F_{r+1}\right. \\
& \left.\quad+\sum_{k=1}^{n-2}\left(\frac{F_{r+2}}{F_{r+1}} \cdot F_{r+1+k}-F_{r+2+k}\right) x^{n-(k+1)}\right] \\
& \quad \times\left(F_{r+1}+F_{r+n+1}\right)^{n-2},
\end{aligned}
$$

where $F_{r+n}$ is the $(r+n)$ th Fibonacci number.

Theorem 12. Let $B_{r, n}^{\prime \prime}=\operatorname{SLCirc}\left(F_{r+1}, \ldots, F_{r+n}\right)$ be a skew left circulant matrix; then, $B_{r, n}^{\prime \prime}$ is an invertible matrix.

Theorem 13. Let $B_{r, n}^{\prime \prime}=\operatorname{SLCirc}\left(F_{r+1}, \ldots, F_{r+n}\right)$ be a skew left circulant matrix; then,

$$
B_{r, n}^{\prime \prime}{ }^{-1}=\frac{1}{f_{n}} \operatorname{SLCirc}\left(x_{1}^{\prime \prime}, x_{2}^{\prime \prime}, \ldots, x_{n}^{\prime \prime}\right) \text {, }
$$


where

$$
\begin{aligned}
x_{1}^{\prime \prime}= & 1-\left(F_{r+3}-s F_{r+2}\right) \cdot \frac{(-a)^{n-3}}{b^{n-2}} \\
& -\sum_{i=1}^{n-3}\left(F_{r+n+2-i}-s F_{r+n+1-i}\right) \cdot \frac{(-a)^{i-1}}{b^{i}}, \\
x_{k}^{\prime \prime}= & -x_{n-k+2}^{\prime} \\
= & \sum_{i=1}^{2}\left(f_{r+1+i}-t f_{r+i}\right) \cdot \frac{(-a)^{n-k-3+i}}{b^{n-k-2+i}}, \\
x_{n-1}^{\prime \prime}= & -x_{3}^{\prime}=\left(F_{r+3}-s F_{r+2}\right) \cdot \frac{1}{b}, \\
x_{n}^{\prime \prime}= & -x_{2}^{\prime} \quad \\
= & s+\sum_{i=1}^{n-2}\left(F_{r+n+1-i}-s F_{r+n-i}\right) \cdot \frac{(-a)^{i-1}}{b^{i}} .
\end{aligned}
$$

\section{Norm and Spread of Skew Left Circulant Matrix with the Fibonacci Numbers}

Theorem 14. Let $B_{r, n}^{\prime \prime}=\operatorname{SLCirc}\left(F_{r+1}, \ldots, F_{r+n}\right)$ be a skew left circulant matrix. Then three kinds of norms of $B_{r, n}^{\prime \prime}$ are given by

$$
\begin{gathered}
\left\|B_{r, n}^{\prime \prime}\right\|_{1}=\left\|B_{r, n}^{\prime \prime}\right\|_{\infty}=F_{r+n+2}-F_{r+2}, \\
\left\|B_{r, n}^{\prime \prime}\right\|_{F}=\sqrt{n\left(F_{r+n} F_{r+n+1}-F_{r} F_{r+1}\right)} .
\end{gathered}
$$

Proof. Using the method in Theorem 8 similarly, the conclusion is obtained.

Theorem 15. Let

$$
B_{r, n}^{\prime \prime \prime}=\operatorname{SLCirc}\left(F_{r+1},-F_{r+2}, \ldots,-F_{r+n-1}, F_{r+n}\right)
$$

be an odd-order alternative skew left circulant matrix; then,

$$
\left\|B_{r, n}^{\prime \prime \prime}\right\|_{2}=\sum_{i=1}^{n} F_{r+i}=F_{r+n+2}-F_{r+2} .
$$

Proof. According to Lemma 4 in [21],

$$
\lambda_{j}\left(B_{r, n}^{\prime \prime \prime}\right)= \pm\left|\sum_{i=1}^{n}(-1)^{i-1} F_{r+i} \omega^{(j-(1 / 2))(k-1)}\right|,
$$

for $j=1,2, \ldots,(n-1) / 2$, and

$$
\lambda_{(n+1) / 2}\left(B_{r, n}^{\prime \prime \prime}\right)=\sum_{i=1}^{n} F_{r+i} .
$$

So

$$
\begin{aligned}
\left|\lambda_{j}\left(B_{r, n}^{\prime \prime \prime}\right)\right| & \leq \sum_{i=1}^{n}\left|(-1)^{i-1} F_{r+i}(-1)^{i-1}\right| \\
& =\sum_{i=1}^{n} F_{r+i}, \quad\left(j=1,2, \cdots, \frac{n+1}{2}\right) .
\end{aligned}
$$

By (55) and (56), we know

$$
\max _{0 \leq i \leq(n+1) / 2}\left|\lambda_{i}\left(B_{r, n}^{\prime \prime \prime}\right)\right|=\sum_{i=1}^{n} F_{r+i} .
$$

Since all skew left circulant matrices are symmetrical, by Lemma 9 in [21], (5), and (57), we obtain

$$
\left\|B_{r, n}^{\prime \prime \prime}\right\|_{2}=F_{r+n+2}-F_{r+2} \text {. }
$$

Theorem 16. Let $B_{r, n}^{\prime \prime}=\operatorname{SLCirc}\left(F_{r+1}, \ldots, F_{r+n}\right)$ be skew left circulant matrix, if $n$ is odd, then

$$
\begin{aligned}
2 F_{r+n} & \leq s\left(B_{r, n}^{\prime \prime}\right) \\
& \leq \sqrt{2 n\left(F_{r+n} F_{r+n+1}-F_{r+1} F_{r}\right)-\frac{2}{n}\left(F_{r+n-1}+F_{r-1}\right)^{2}} ;
\end{aligned}
$$

if $n$ is even, then

$$
2 F_{r+n} \leq s\left(B_{r, n}^{\prime \prime}\right) \leqslant \sqrt{2 n\left(F_{r+n} F_{r+n+1}-F_{r+1} F_{r}\right)} .
$$

Proof. Since $B_{r, n}^{\prime \prime}$ is a symmetric matrix, by (20) in [21],

$$
s\left(B_{r, n}^{\prime \prime}\right) \geq 2 \max _{i \neq j}\left|a_{i j}\right|=2 F_{r+n} .
$$

If $n$ is odd, the trace of $B_{r, n}^{\prime \prime}$ is

$$
\begin{aligned}
\operatorname{tr} & \left(B_{r, n}^{\prime \prime}\right) \\
& =F_{r+1}-F_{r+2}+F_{r+3}-\cdots+F_{r+n} \\
& =F_{r+1}+F_{r+1}+F_{r+3}+\cdots+F_{r+n-2} \\
& =2 F_{r+1}+F_{r+1}+F_{r+2}+\cdots+F_{r+n-3} \\
& =2 F_{r+1}+\sum_{i=1}^{n-3} F_{r+i} ;
\end{aligned}
$$

by (5), we know

$$
\operatorname{tr}\left(B_{r, n}^{\prime \prime}\right)=F_{r+n-1}+F_{r-1} .
$$

By (18) in [21], (51), and (63), we obtain

$$
\begin{aligned}
& s\left(B_{r, n}^{\prime \prime}\right) \\
& \leqslant \sqrt{2 n\left(F_{r+n} F_{r+n+1}-F_{r} F_{r+1}\right)-\frac{2}{n}\left(F_{r+n-1}+F_{r-1}\right)^{2}} .
\end{aligned}
$$

If $n$ is even, the trace of $B_{r, n}^{\prime \prime}$ is

$$
\begin{aligned}
\operatorname{tr}\left(B_{r, n}^{\prime \prime}\right)= & F_{r+1}-F_{r+1}+F_{r+3} \\
& -F_{r+3} \cdots+F_{r+n-1}-F_{r+n-1}=0 .
\end{aligned}
$$

By (18) in [21], (51), and (65), we can get

$$
s\left(B_{r, n}^{\prime \prime}\right) \leqslant \sqrt{2 n\left(F_{r+n} F_{r+n+1}-F_{r} F_{r+1}\right)} .
$$

So the result follows. 


\section{Conclusion}

We discuss the invertibility of skew circulant type matrices with any continuous Fibonacci numbers and present the determinant and the inverse matrices by constructing the transformation matrices. The four kinds of norms and bounds for the spread of these matrices are given, respectively. In [20], a new fast algorithm for optimal design of block digital filters (BDFs) was proposed based on skew circulant matrix. The reason why we focus our attentions on skew circulant is to explore the application of skew circulant in the related field in real-time tracking and networks engineering. On the basis of method of [17] and ideas of [43], we will exploit real-time tracking with kernel matrix of skew circulant structure. On the basis of existing application situation [1-11], we will exploit application of network engineering based on skew circulant matrix.

\section{Conflict of Interests}

The authors declare that there is no conflict of interests regarding the publication of this paper.

\section{Acknowledgments}

The research was supported by the Development Project of Science and Technology of Shandong Province (Grant no. 2012GGX10115) and NSFC (Grant no. 11301251) and the AMEP of Linyi University, China.

\section{References}

[1] M. P. Joy and V. Tavsanoglu, "Circulant matrices and the stability of a class of CNNs," International Journal of Circuit Theory and Applications, vol. 24, no. 1, pp. 7-13, 1996.

[2] D. Rocchesso and J. O. Smith, "Circulant and elliptic feedback delay networks for artificial reverberation," IEEE Transactions on Speech and Audio Processing, vol. 5, no. 1, pp. 51-63, 1997.

[3] Y. Jing and H. Jafarkhani, "Distributed differential space-time coding for wireless relay networks," IEEE Transactions on Communications, vol. 56, no. 7, pp. 1092-1100, 2008.

[4] H. Eghbali, S. Muhaidat, S. A. Hejazi, and Y. Ding, "Relay selection strategies for single-carrier frequency-domain equalization multi-relay cooperative networks," IEEE Transactions on Wireless Communications, vol. 12, no. 5, pp. 2034-2045, 2013.

[5] D. Rocchesso, "Maximally diffusive yet efficient feedback delay networks for artificial reverberation," IEEE Signal Processing Letters, vol. 4, no. 9, pp. 252-255, 1997.

[6] S. Sardellitti, S. Barbarossa, and A. Swami, "Optimal topology control and power allocation for minimum energy consumption in consensus networks," IEEE Transactions on Signal Processing, vol. 60, no. 1, pp. 383-399, 2012.

[7] J. Li, J. Yuan, R. Malaney, M. Xiao, and W. Chen, "Fulldiversity binary frame-wise network coding for multiplesource multiple-relay networks over slow-fading channels," IEEE Transactions on Vehicular Technology, vol. 61, no. 3, pp. 1346-1360, 2012.

[8] W. Hirt and J. L. Massey, "Capacity of the discrete-time Gaussian channel with intersymbol interference," IEEE Transactions on Information Theory, vol. 34, no. 3, pp. 380-388, 1988.
[9] H. Wang, X.-G. Xia, and Q. Yin, "Distributed space-frequency codes for cooperative communication systems with multiple carrier frequency offsets," IEEE Transactions on Wireless Communications, vol. 8, no. 2, pp. 1045-1055, 2009.

[10] F. Gao, B. Jiang, X. Gao, and X.-D. Zhang, "Superimposed training based channel estimation for OFDM modulated amplifyand-forward relay networks," IEEE Transactions on Communications, vol. 59, no. 7, pp. 2029-2039, 2011.

[11] G. Wang, F. Gao, Y.-C. Wu, and C. Tellambura, "Joint CFO and channel estimation for OFDM-based two-way relay networks," IEEE Transactions on Wireless Communications, vol. 10, no. 2, pp. 456-465, 2011.

[12] D. Bertaccini and M. K. Ng, "Skew-circulant preconditioners for systems of LMF-based ODE codes," in Numerical Analysis and Its Applications, vol. 1988 of Lecture Notes in Computer Science, pp. 93-101, 2001.

[13] R. H. Chan and X.-Q. Jin, "Circulant and skew-circulant preconditioners for skew-Hermitian type Toeplitz systems," BIT, vol. 31, no. 4, pp. 632-646, 1991.

[14] R. H. Chan and M.-K. Ng, "Toeplitz preconditioners for Hermitian Toeplitz systems," Linear Algebra and Its Applications, vol. 190, pp. 181-208, 1993.

[15] T. Huckle, "Circulant and skewcirculant matrices for solving Toeplitz matrix problems," SIAM Journal on Matrix Analysis and Applications, vol. 13, no. 3, pp. 767-777, 1992.

[16] J. N. Lyness and T. Sørevik, "Four-dimensional lattice rules generated by skew-circulant matrices," Mathematics of Computation, vol. 73, no. 245, pp. 279-295, 2004.

[17] M. J. Narasimha, "Linear convolution using skew-cyclic convolutions," IEEE Signal Processing Letters, vol. 14, no. 3, pp. 173-176, 2007.

[18] V. C. Liu and P. P. Vaidyanathan, "Circulant and skew-circulant matrices as new normal-form realization of IIR digital filters," IEEE Transactions on Circuits and Systems, vol. 35, no. 6, pp. 625-635, 1988

[19] J. Li, Z. Jiang, N. Shen, and J. Zhou, "On optimal backward perturbation analysis for the linear system with skew circulant coefficient matrix," Computational and Mathematical Methods in Medicine, vol. 2013, Article ID 707381, 7 pages, 2013.

[20] D. Q. Fu, Z. L. Jiang, Y. F. Cui, and S. T. Jhang, "A new fast algorithm for optimal design of block digital filters by skewcyclic convolution," IET Signal Processing, 6 pages, 2014.

[21] J.-J. Yao and Z.-L. Jiang, “The determinants, inverses, norm, and spread of skew circulant type matrices involving any continuous lucas numbers," Journal of Applied Mathematics, vol. 2014, Article ID 239693, 10 pages, 2014.

[22] S.-Q. Shen, J.-M. Cen, and Y. Hao, "On the determinants and inverses of circulant matrices with Fibonacci and Lucas numbers," Applied Mathematics and Computation, vol. 217, no. 23, pp. 9790-9797, 2011.

[23] Y. Gao, Z. Jiang, and Y. Gong, "On the determinants and inverses of skew circulant and skew left circulant matrices with Fibonacci and Lucas numbers," WSEAS Transactions on Mathematics, vol. 12, no. 4, pp. 472-481, 2013.

[24] X.-Y. Jiang, Y. Gao, and Z.-L. Jiang, "Determinants and inverses of skew and skew left circulant matrices involving the $k$ Fibonacci numbers in communications-I," Far East Journal of Mathematical Sciences, vol. 76, no. 1, pp. 123-137, 2013.

[25] X.-Y. Jiang, Y. Gao, and Z.-L. Jiang, "Determinants and inverses of skew and skew left circulant matrices involving the $k$ Lucas numbers in communications-II," Far East Journal of Mathematical Sciences, vol. 78, no. 1, pp. 1-17, 2013. 
[26] D. V. Jaiswal, "On determinants involving generalized Fibonacci numbers," The Fibonacci Quarterly, vol. 7, pp. 319-330, 1969.

[27] D. A. Lind, "A Fibonacci circulant," The Fibonacci Quarterly, vol. 8, no. 5, pp. 449-455, 1970.

[28] L. Dazheng, "Fibonacci-Lucas quasi-cyclic matrices," The Fibonacci Quarterly, vol. 40, no. 3, pp. 280-286, 2002.

[29] S. Solak, "On the norms of circulant matrices with the Fibonacci and Lucas numbers," Applied Mathematics and Computation, vol. 160, no. 1, pp. 125-132, 2005.

[30] A. İpek, "On the spectral norms of circulant matrices with classical Fibonacci and Lucas numbers entries," Applied Mathematics and Computation, vol. 217, no. 12, pp. 6011-6012, 2011.

[31] S. Shen and J. Cen, "On the bounds for the norms of $r$ circulant matrices with the Fibonacci and Lucas numbers," Applied Mathematics and Computation, vol. 216, no. 10, pp. 2891-2897, 2010.

[32] M. Akbulak and D. Bozkurt, "On the norms of Toeplitz matrices involving Fibonacci and Lucas numbers," Hacettepe Journal of Mathematics and Statistics, vol. 37, no. 2, pp. 89-95, 2008.

[33] A. Bose, R. S. Hazra, and K. Saha, "Spectral norm of circulanttype matrices," Journal of Theoretical Probability, vol. 24, no. 2, pp. 479-516, 2011.

[34] L. Mirsky, "The spread of a matrix," Mathematika, vol. 3, pp. 127130, 1956.

[35] R. Sharma and R. Kumar, "Remark on upper bounds for the spread of a matrix," Linear Algebra and Its Applications, vol. 438, no. 11, pp. 4359-4362, 2013.

[36] J. Wu, P. Zhang, and W. Liao, "Upper bounds for the spread of a matrix," Linear Algebra and Its Applications, vol. 437, no. 11, pp. 2813-2822, 2012.

[37] C. R. Johnson, R. Kumar, and H. Wolkowicz, "Lower bounds for the spread of a matrix," Linear Algebra and Its Applications, vol. 29, pp. 161-173, 1985.

[38] V. W. de Spinadel, "The metallic means and design," in Nexus II: Architecture and Mathematics, K. Williams, Ed., vol. 5, pp. 143-157, Edizioni dell'Erba, 1998.

[39] V. W. de Spinadel, "The metallic means family and forbidden symmetries," International Mathematical Journal, vol. 2, no. 3, pp. 279-288, 2002.

[40] M. S. El Naschie, "Modular groups in Cantorian $\mathrm{E}_{\infty}$ highenergy physics," Chaos, Solitons \& Fractals, vol. 16, no. 2, pp. 353-366, 2003.

[41] S. Falcón and Á. Plaza, "The $k$-Fibonacci hyperbolic functions," Chaos, Solitons \& Fractals, vol. 38, no. 2, pp. 409-420, 2008.

[42] A. P. Stakhov and I. S. Tkachenko, "Hyperbolic trigonometry of Fibonacci," Reports of the National Academy of Sciences of Ukraine, vol. 208, no. 7, pp. 9-14, 1993 (Russian).

[43] J. F. Henriques, R. Caseiro, P. Martins, and J. Batista, "Exploiting the circulant structure of tracking-by-detection with kernels," in Proceedings of the European Conference on Computer Vision (ECCV '12), pp. 1-14, 2012. 


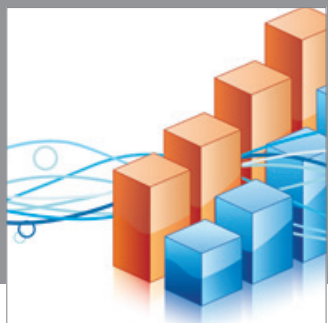

Advances in

Operations Research

mansans

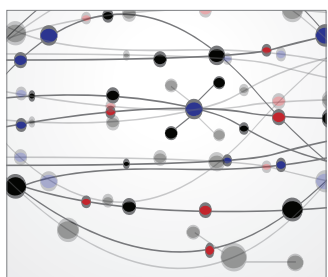

The Scientific World Journal
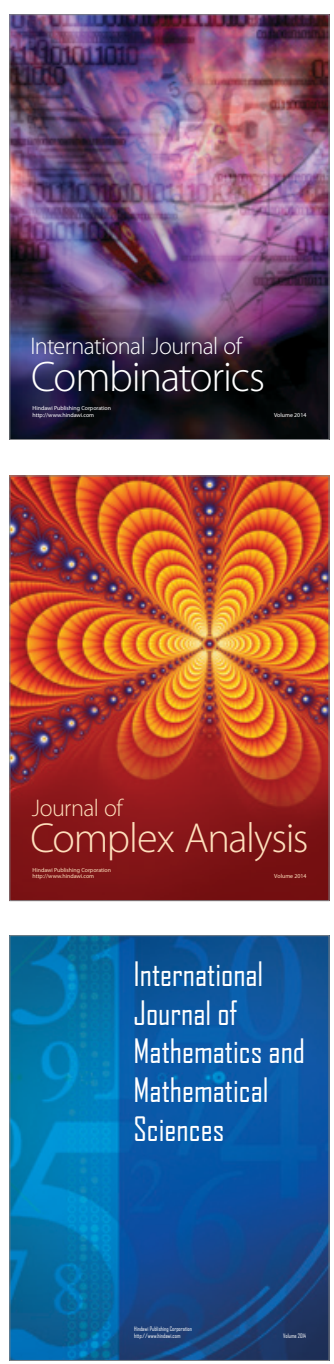
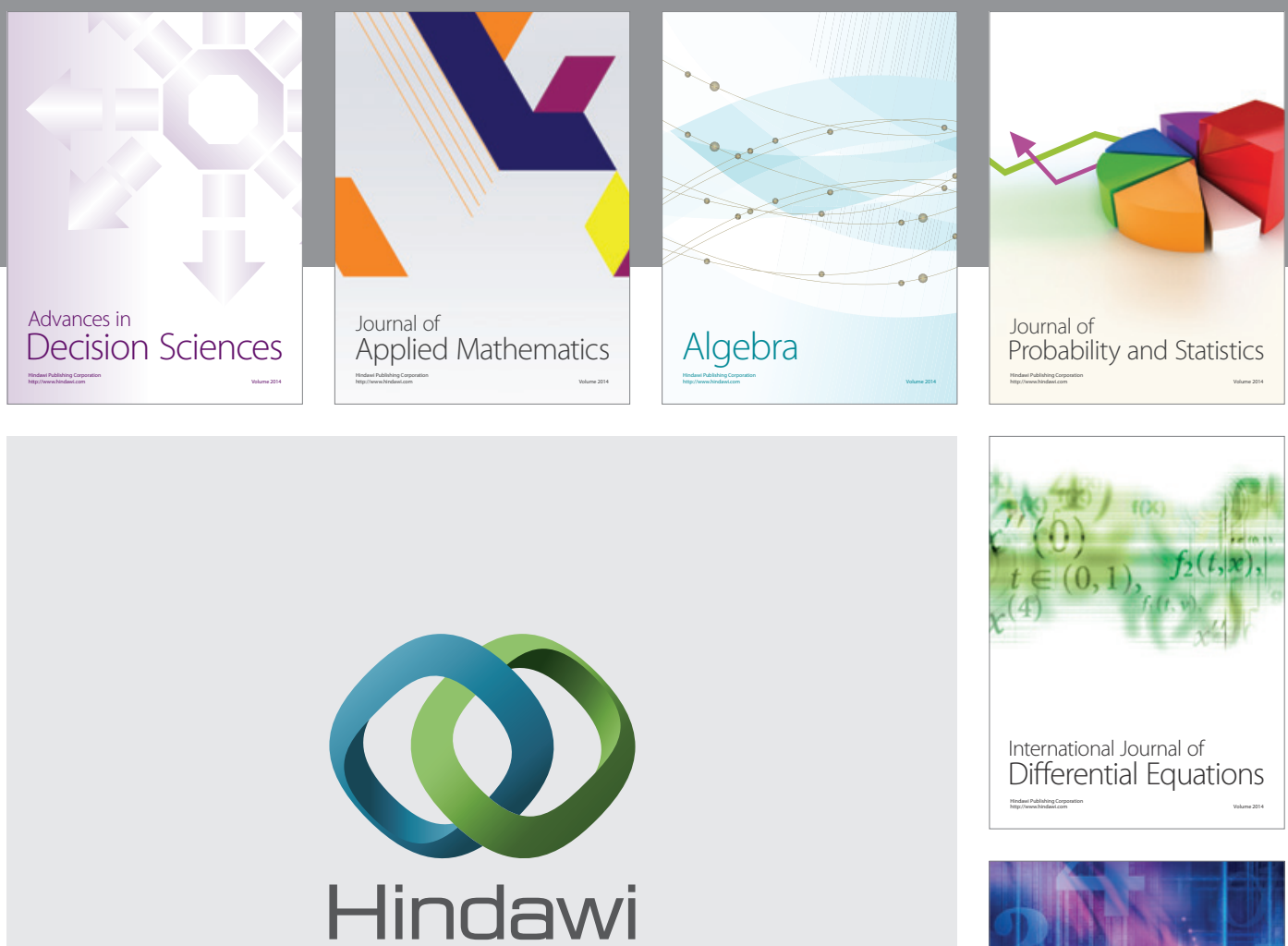

Submit your manuscripts at http://www.hindawi.com
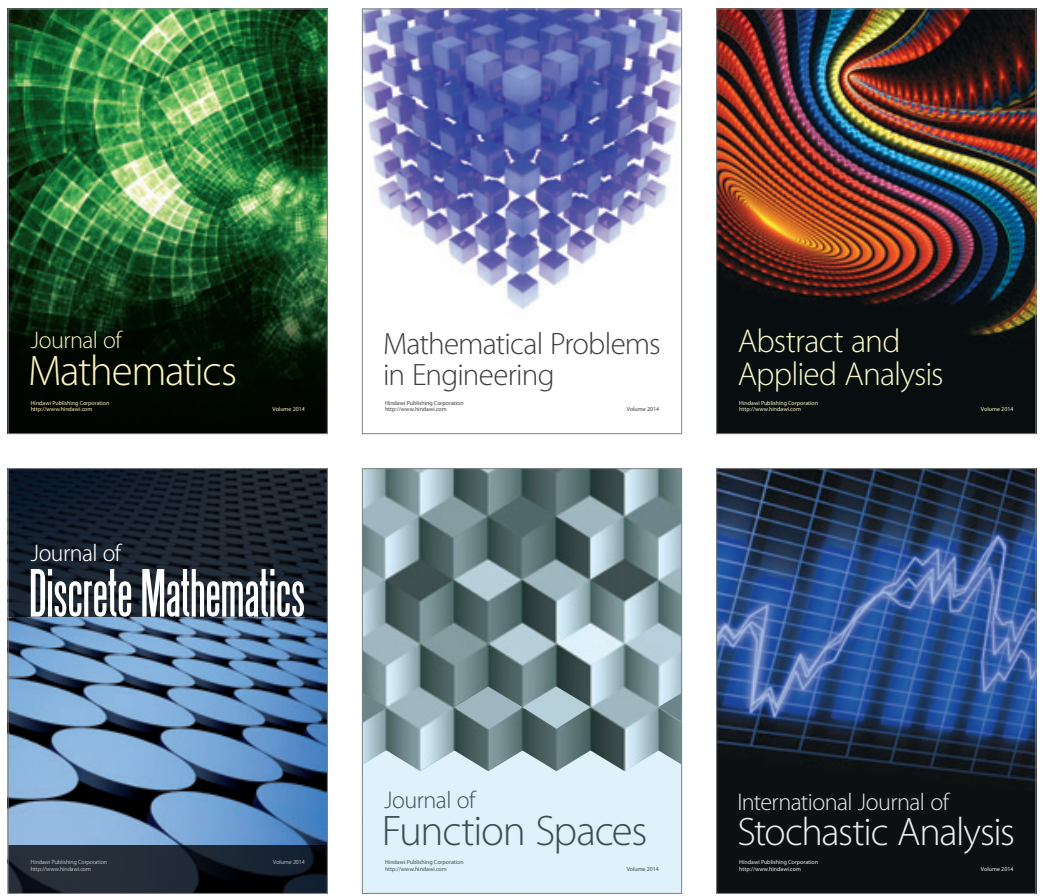

Journal of

Function Spaces

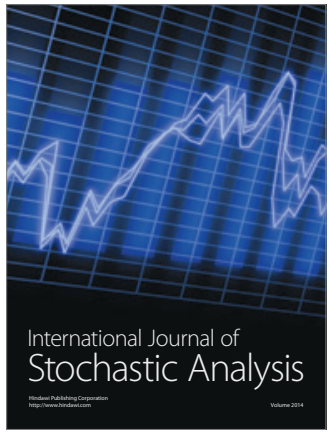

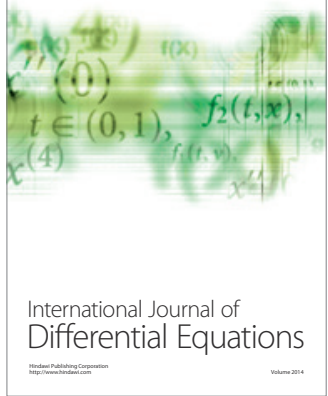
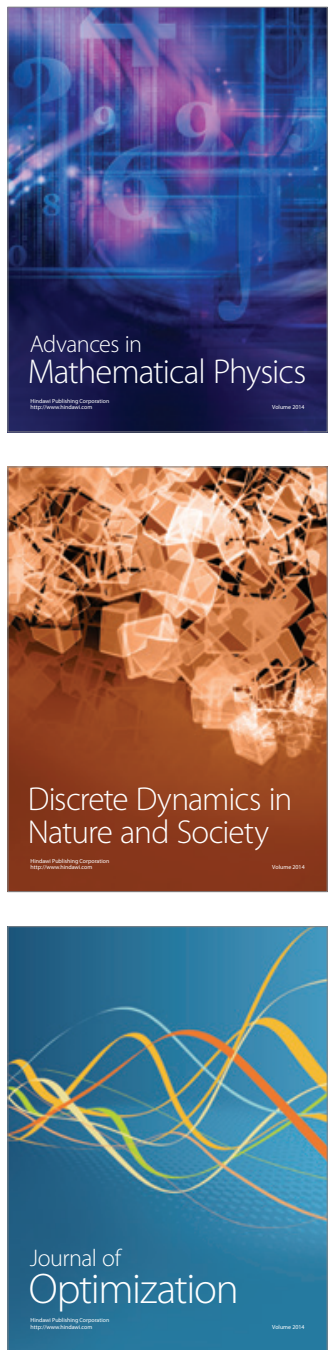